\title{
Different resistance of bread wheat near-isogenic lines carrying various combinations of alleles of the $P p$ and $B a$ genes regulating the biosynthesis of anthocyanin to the pathogenic fungus Stagonospora nodorum
}

\author{
Veselova S.V. ${ }^{1 *}$, Shoeva O.Y. ${ }^{2}$, Nuzhnaya T.V. ${ }^{1}$, Burkhanova G.F. ${ }^{1}$, Rumyantsev S.D. ${ }^{1}$ \\ ${ }^{1}$ Institute of Biochemistry and Genetics of the UFRC of the RAS, Ufa, Russia \\ ${ }^{2}$ Institute of Cytology and Genetics, SB RAS, Novosibirsk, Russia \\ * email: veselova75@rambler.ru
}

In recent years, there has been an increasing interest in the production of wheat, which accumulates anthocyanin compounds in grain, as a source of useful food products. However, the significance of these substances for wheat plant protection has not been sufficiently estimated yet. We used a set of nearly isogenic (NILs) and substitution lines carrying various combinations of dominant and recessive alleles of the $P p$ and $B a$ genes, which regulate the biosynthesis of anthocyanins in the pericarp/coleoptile and aleurone layer respectively. The lines have been developed in the genetic background of spring bread wheat (Triticum aestivum L.) Saratovskaya 29 (S29). Variety S29 and the lines were infected with two isolates of the pathogenic fungus Stagonospora nodorum carrying a different set of necrotrophic effectors Sn9MN (ToxA/Tox3/Tox1) and Sn1SP (Tox1). We found the Snnl susceptibility gene to the $S$. nodorum effector SnTox1 in variety S29 and the lines. The variety S29 showed susceptibility to the Sn9MN isolate and resistance to the Sn1SP isolate. Lines iP2A, iP7D, iP were more susceptible to both isolates of the pathogen than S29. Lines iJP7A, iPF7D and substitution line s:S29Ba, were more resistant to both isolates of the pathogen than S29. The lines iPF2A, iPF showed the greatest resistance among the other lines. A gain in the resistance of each individual line was accompanied by intensification in the generation of hydrogen peroxide, an increase in the activity of peroxidases, and a change in the expression of the Snnl gene at an early stage of infection with the pathogen (up to 3 days). Such a different degree of NILs resistance to $S$. nodorum may be due to differences in the composition of introgressed genomics fragments.

Acknowledgments: This work was supported by the RFBR project No. 20-316-80047. 